ОПТИМИЗАЦИЯ КОНСТРУКЦИИ ИЗМЕРИТЕЛЬНОГО ПРЕОБРАЗОВАТЕЛЯ ПРИ ОПРЕДЕЛЕНИИ НАПРЯЖЕННОСТИ МАГНИТНОГО ПОЛЯ НА ПОВЕРХНОСТИ ИСПЫТУЕМОГО ОБРАЗЦА

\author{
Кучер А.И. ${ }^{1}$, Ланкин М.В. ${ }^{1}$ \\ ${ }^{1}$ ФБОУ ВПО «Южно-Российский Государственный Политехнический Университет (НПИ) имени М.И. Пла- \\ това», Новочеркасск, Россия (346428, Новочеркасск, ул. Просвещения, 132), e-mail: artiom.kucher@gmail.com \\ Предложен метод измерения напряженности магнитного поля на поверхности испытуемого образца при \\ испытании листовой электротехнической стали с помощью устройства экспресс-контроля изделий из \\ листовой электротехнической стали. Метод основан на линейно аппроксимации характеристики и за- \\ ключается в определении напряженности магнитного поля в двух точках на известном удалении от ис- \\ пытуемого образца и экстраполяции полученной зависимости на поверхность испытуемого образца. Для \\ данного метода проведена оптимизация координат измерения напряженности магнитного поля методом \\ крутого восхождения для минимизации погрешности экстраполяции. Оптимизация позволила умень- \\ шить погрешность определения напряженности магнитного поля на поверхности испытуемого образца. с \\ $8 \%$ до $0.32 \%$. Для проведения измерения построена конечно-элементная модель накладного датчика \\ устройства экспресс-контроля изделий из листовой электротехнической стали в среде ANSYS Maxwell. \\ Ключевые слова: оптимизация, метод крутого восхождения, испытание электротехнической стали
}

\title{
OPTIMIZATION OF TRANSMITTER CONSTRUCTION IN DETERMENING OF MAGNETIC FIELD STRENGTH ON SURFACE OF THE TEST SAMPLE
}

\begin{abstract}
Kucher A.I. ${ }^{1}$, Lankin M.V. ${ }^{1}$
${ }^{1}$ South-Russia State Polytechnic University (NPI) n.a. M.I. Platov, Novocherkassk, Russia (346428, Novocherkassk, Prosveshenia str., 132), e-mail: artiom.kucher@gmail.com

We have proposed a method for measuring of magnetic field strength at the surface of the test sample when testing electrical steel with using device for express-control of electrical steel products. The method is based on a linear approximation of the characteristics and consists in the definition of the magnetic field at two points at a certain distance from the test sample and the extrapolation obtained depending on the surface of the test sample. For this method we have done optimization of testing points coordinates with using method of rapid progress for minimization of extrapolation error. Optimization allowed to reduce the error in the determination of the magnetic field on the surface of the test specimen. from $8 \%$ to $0.32 \%$. To measure constructed finite element model on sensor devices express control of products from electrical steel sheet in ANSYS Maxwell.
\end{abstract}

Keywords: optimization, method of rapid progress, testing of electrical steel

При производстве деталей и изделий из листовой электротехнической стали необходимо уделять особое внимание контролю магнитных характеристик материала, так как эти характеристики зависят от способа обработки. Помимо этого, необходимость такого контроля обусловлена получением готовой продукции (например электродвигателей) с заданными эксплуатационными параметрами. Промежуточный контроль магнитных характеристик (в частности $B(H)$-характеристики) стали и изделий на ее основе делает технологический процесс гибким и минимизирует процент брака готовых электротехнических изделий.

Известно устройство [3]. Данное устройство позволяет получить вебер-амперную и $B(H)$-характеристику характеристику участка детали из листовой электротехнической стали. Напряженность магнитного поля в образце определяется косвенным образом по формуле: 


$$
H=\frac{I \cdot W}{\Delta},
$$

где $I$ - ток, протекающей в намагничивающей обмотке; $W$ - число витков намагничивающей катушки; $\Delta$ - величина воздушного зазора, в который помещен образец.

Величина $\Delta$ не постоянна, так как после входа в насыщение образца фактическое значение зазора возрастает за счет насыщения сердечника намагничивающей системы в районе контакта с образцом. Из-за этого возникает погрешность измерения напряженности магнитного поля на поверхности листа электротехнической стали. Для компенсации данной погрешности предлагается ввести канал измерения напряженности на поверхности испытуемой детали, представляющий собой линейку из $n$-датчиков Холла, расположенных на некоторых расстояниях $x_{1} \ldots x_{n}$ от листа электротехнической стали. Для определения числа датчиков и их местоположения необходимо построить модель топографии магнитного поля накладного датчика в окрестности испытуемого образца[2].

Для проведения измерений была построена модель накладного датчика. Моделирование выполнено при помощи программы ANSYS Maxwell. ANSYS Maxwell - высокопроизводительное интерактивное программное обеспечение, которое использует конечноэлементный анализ для решения электрических, магнитостатических задач, переходных задач и задач вихревых токов.

Трехмерная конечно-элементная модель представлена на рисунке 1 , где 1 - испытуемый образец; 2 - магнитопровод; 3 - намагничивающие катушки. Отметим, что используя две плоскости симметрии, моделируется 1/4 датчика с целью уменьшения размерности модели и уменьшения времени расчета. Используя плоскость симметрии, моделируется 1/4 дифференциального магнитного моста с целью уменьшения размерности модели и времени расчета.

Измерения проводились вдоль оси $O z$ на отрезке [0,10] мм. Толщина листа стали 0,8 мм. Измерения проведены при различных значениях тока в намагничивающей обмотке. Результаты измерения представлены на рисунке 2.

Как видно из рисунка 2, зависимость напряженности магнитного $H$ поля от расстояния до образца $x$ носит практически линейный характер. Таким образом, для определения напряженности магнитного поля на поверхности испытуемого образца достаточно измерения напряженности $H_{1}$ и $H_{2}$ в двух точках пространства $x_{1}$ и $x_{2}$ и экстраполировании полученной зависимости в точку $x=0$ (рисунок 3 ). 


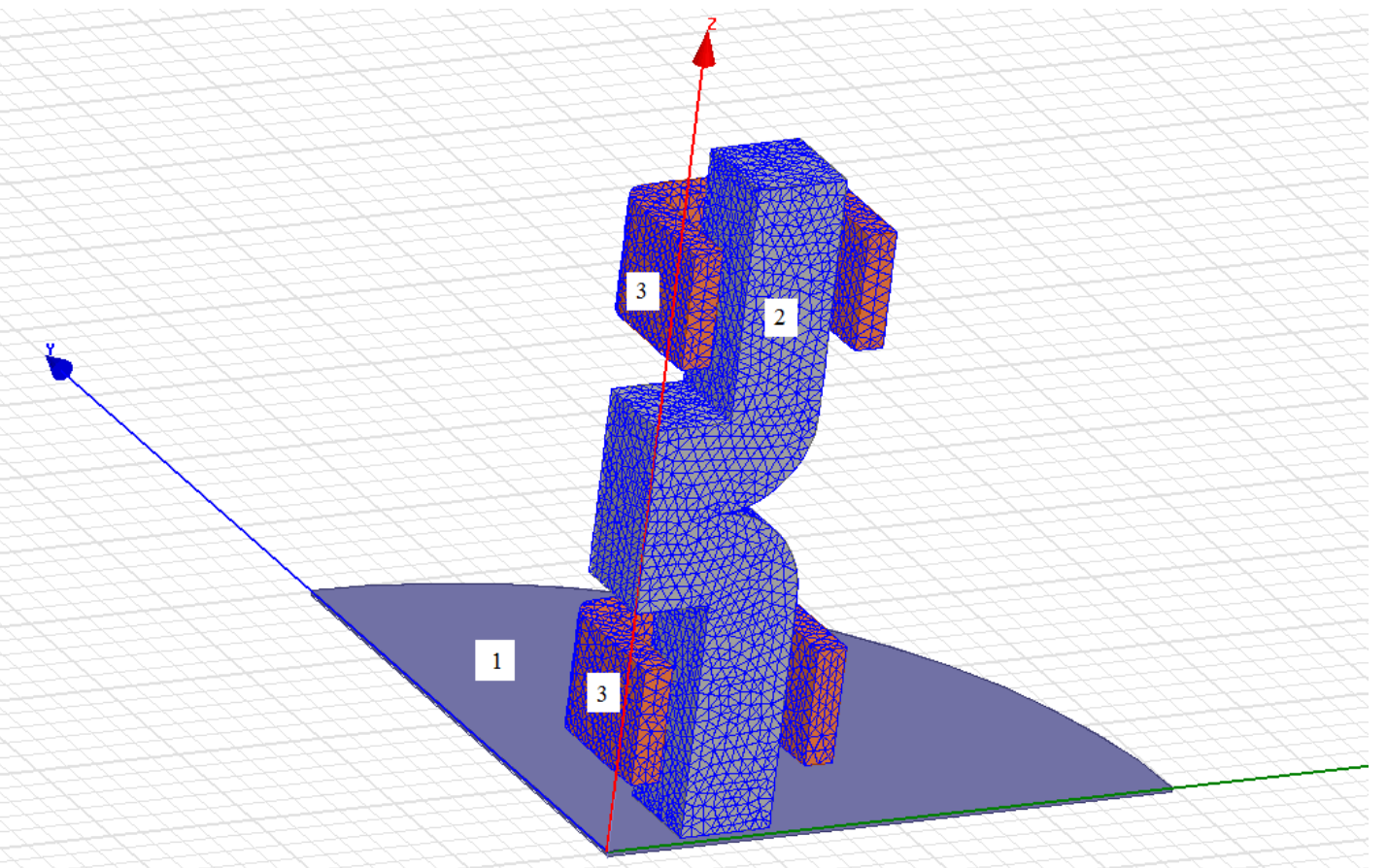

Рис. 1. Конечно-элементная модель накладного датчика

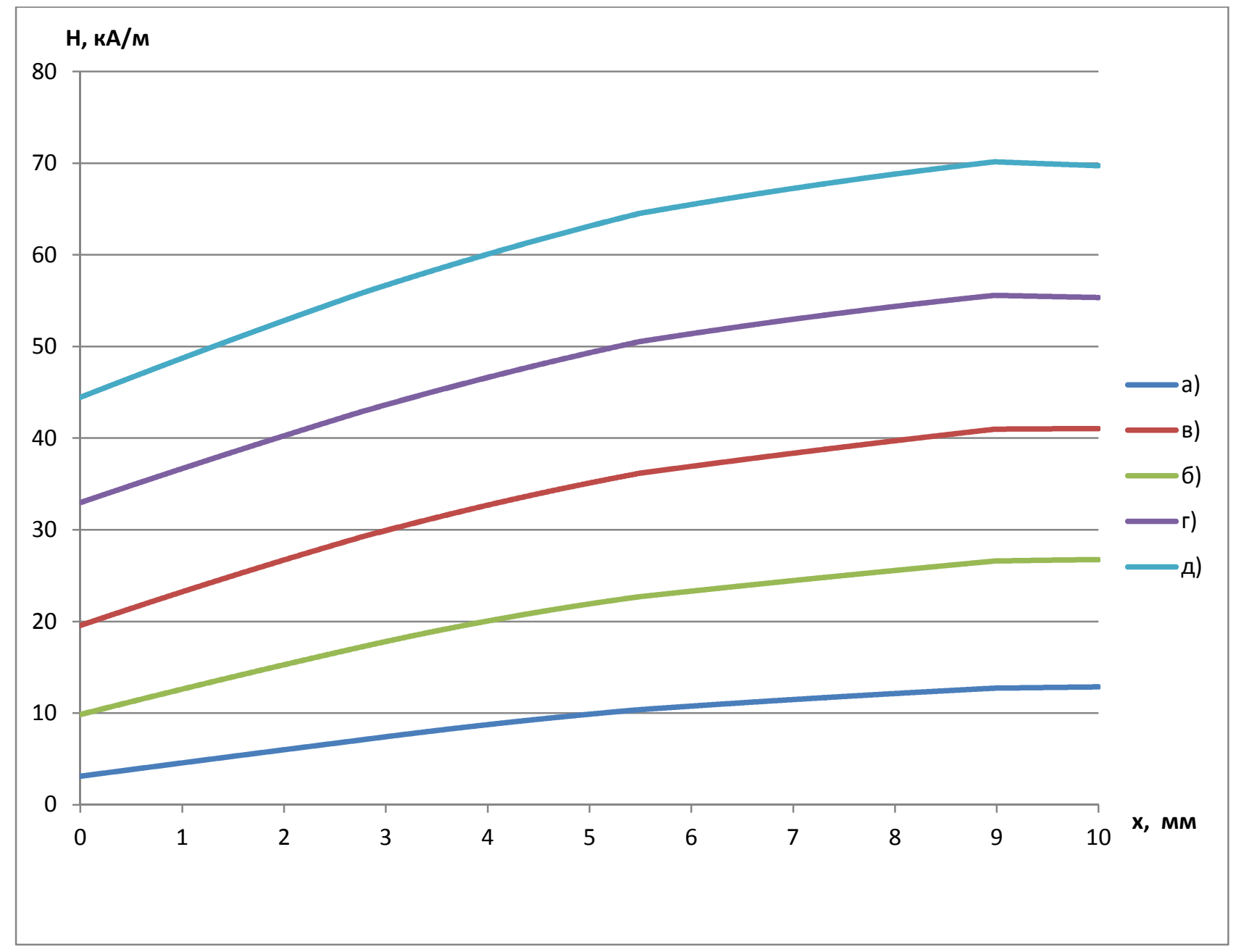

Рис.2. Результаты измерения при различном числе ампер-витков:

а) 200 ; б) 400 ; в) 600; г) 800 ; д) 1000 . 


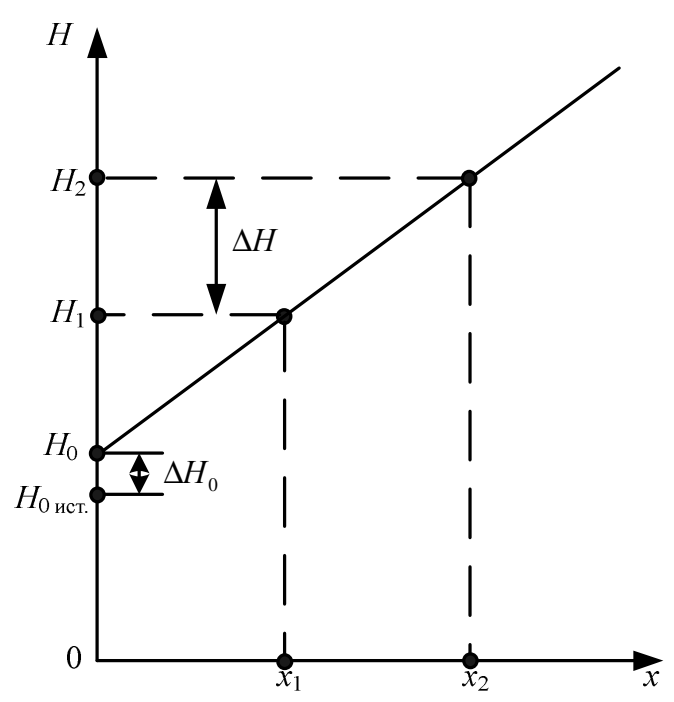

Рис.3. Определения напряженности МП на поверхности испытуемого образца при использовании линейной экстраполяции

Расчет напряженности МП на поверхности ПМ производится по формуле [22]:

$$
\begin{gathered}
H_{0}=\frac{H_{2} \cdot x_{1}-H_{1} \cdot x_{2}}{x_{1}-x_{2}} . \\
H_{0}=A_{1} \cdot H_{1}+A_{2} \cdot H_{2}, \\
A_{1}=\frac{x_{1}}{x_{1}-x_{2}} ; A_{2}=\frac{-x_{2}}{x_{1}-x_{2}}
\end{gathered}
$$

Таким образом, для нахождения значения напряженности магнитного поля на поверхности испытуемого образца достаточно знать значение напряженности магнитного поля в двух фиксированных точка на некотором удалении от поверхности.

Для оптимизации координат измерения напряженности магнитного поля воспользуемся методом крутого восхождения [20]. Метод крутого восхождения позволяет попасть из произвольной точки области исследования в область экстремума, поэтому выбор начальной точки не влияет на конечный результат. Задавшись произвольными координатами $X_{i 0}$ начальной точки $O_{i}$, строим для области, прилегающей к ней, эмпирическую модель первого порядка:

$$
Y=B_{0}+B_{1} X_{1}+B_{2} X_{2}
$$

Где: $B_{i}$-значения коэффициентов уравнения; $X_{i}$ - факторы; $Y$ - отклик.

Зададимся центром плана для всех факторов и интервалом варьирования исходя из априорной информации. Произведем кодирование факторов по формуле:

$$
X_{i}=\frac{x_{i}-x_{i 0}}{h_{i}} ; i=1,2, \ldots, n \text {. }
$$


Для движения по методу крутого восхождения необходимо построить модель первого порядка для начальных координат. По полученному уравнению регрессии определяется градиент и производится движение поэтому градиенту до точки экстремума. Матрица планирования эксперимента $2^{2}$ для нахождения уравнения регрессии первого порядка приведена в таблице 1. Обработка полученных результатов произведена посредством программного средства Statistica 6.0. Для анализа полученных результатов и проверки адекватности модели необходимо задаться числом повторных опытов $m=3$. Зададимся погрешностью расчета значения погрешности $1 \%$.

Таблица 1

Матрица планирования эксперимента

\begin{tabular}{|c|c|c|c|c|c|c|}
\hline $\begin{array}{c}\text { № } \\
\text { опыта }\end{array}$ & $X 1$ & $\begin{array}{c}x_{1}, \\
\mathcal{M M}\end{array}$ & $X 2$ & $\begin{array}{c}x_{2}, \\
\mathcal{M M}\end{array}$ & $\begin{array}{c}H_{0}, \\
\text { кA/M }\end{array}$ & $\begin{array}{c}\delta, \\
\%\end{array}$ \\
\hline 1 & 2 & 3 & 4 & 5 & 6 & 7 \\
\hline 1 & + & 2.5 & + & 4.5 & 6.97 & 14.09 \\
\hline 2 & + & 2.5 & + & 4.5 & 7.04 & 15.23 \\
\hline 3 & + & 2.5 & + & 4.5 & 6.90 & 12.95 \\
\hline 4 & - & 1.5 & + & 4.5 & 6.47 & 6.00 \\
\hline 5 & - & 1.5 & + & 4.5 & 6.54 & 7.06 \\
\hline 6 & - & 1.5 & + & 4.5 & 6.41 & 4.94 \\
\hline 7 & + & 2.5 & - & 3.5 & 6.78 & 10.98 \\
\hline 8 & + & 2.5 & - & 3.5 & 6.85 & 12.09 \\
\hline 9 & + & 2.5 & - & 3.5 & 6.71 & 9.87 \\
\hline 10 & - & 1.5 & - & 3.5 & 6.33 & 3.72 \\
\hline 11 & - & 1.5 & - & 3.5 & 6.40 & 4.76 \\
\hline 12 & - & 1.5 & - & 3.5 & 6.27 & 2.68 \\
\hline
\end{tabular}

В результате получена адекватная модель со всеми значащими коэффициентами регрессии. Уравнение регрессии для кодированных значений уровней факторов имеет вид:

$$
Y=8,698+7,675 X_{1}+2,699 X_{2} \text {. }
$$

Градиент имеет вид:

$$
\operatorname{grad} Y=7,675 i+2,699 j .
$$

Произведем движение по градиенту к указанным координатам. Исходя из центра плана, интервала варьирования факторов и их граничных значений получаем шаг для первого фактора 0,2 мм, для второго фактора 0,4 мм. Движение по градиенту сведено в таблицу 2.

Точка экстремума не найдена, но мы пришли к границе допустимых значений. Вид поверхности отклика и значение градиента указывает, что минимум погрешности будет при максимально близких к поверхности координатам точек измерения. Из физических ограничений размера датчика мы не можем приблизить точки измерения ближе чем $x_{1}=1$ мм и $x_{2}=2$ мм. Исследуем поверхность отклика в районе полученных координат. 
Таблица 2

Движение по градиенту

\begin{tabular}{|c|c|c|c|}
\hline № шага & $x_{1}, \mathrm{MM}$ & $x_{2}$, MM & $\delta, \%$ \\
\hline 1 шаг & 1.8 & 3.6 & 5.43 \\
\hline 2 шаг & 1.6 & 3.2 & 3.06 \\
\hline 3 шаг & 1.4 & 2.8 & 0.89 \\
\hline 4 шаг & 1.2 & 2.4 & 0.47 \\
\hline 5 шаг & 1 & 2 & 0.32 \\
\hline
\end{tabular}

Матрица планирования эксперимента $2^{2}$ для нахождения уравнения регрессии первого порядка приведена в таблице 3.

Таблица 3

Матрица планирования эксперимента

\begin{tabular}{|c|c|l|l|l|l|l|}
\hline $\begin{array}{c}\text { № } \\
\text { опыта }\end{array}$ & $X 1$ & $\begin{array}{c}x_{1}, \\
\text { мM }\end{array}$ & \multicolumn{1}{|c|}{$X 2$} & $\begin{array}{c}x_{2}, \\
\text { мM }\end{array}$ & $\begin{array}{c}H_{0}, \\
\text { кA/M }\end{array}$ & $\begin{array}{c}\delta, \\
\%\end{array}$ \\
\hline 1 & 2 & 3 & 4 & 5 & 6 & 7 \\
\hline 1 & + & 1.4 & + & 2.4 & 6.141 & 0.545 \\
\hline 2 & + & 1.4 & + & 2.4 & 6.144 & 0.596 \\
\hline 3 & + & 1.4 & + & 2.4 & 6.138 & 0.495 \\
\hline 4 & - & 0.6 & + & 2.4 & 6.122 & 0.234 \\
\hline 5 & - & 0.6 & + & 2.4 & 6.125 & 0.284 \\
\hline 6 & - & 0.6 & + & 2.4 & 6.119 & 0.184 \\
\hline 7 & + & 1.4 & - & 1.6 & 6.130 & 0.364 \\
\hline 8 & + & 1.4 & - & 1.6 & 6.133 & 0.414 \\
\hline 9 & + & 1.4 & - & 1.6 & 6.127 & 0.313 \\
\hline 10 & - & 0.6 & - & 1.6 & 6.117 & 0.156 \\
\hline 11 & - & 0.6 & - & 1.6 & 6.120 & 0.206 \\
\hline 12 & - & 0.6 & - & 1.6 & 6.114 & 0.106 \\
\hline
\end{tabular}

В результате получена адекватная модель со всеми значащими коэффициентами регрессии. Уравнение регрессии для некодированных значений уровней факторов имеет вид:

$$
Y=-0,324+0,324 X_{1}+0,162 X_{2} \text {. }
$$

Изменение погрешности при движении показано на рисунке 5.

В результате оптимизации найдены координаты точек измерения, обеспечивающие минимальную погрешность при линейной экстраполяции значения напряженности магнитного поля на поверхности испытуемого образца. Т.к. оптимизация проводилась при среднем значении тока в намагничивающей обмотке, авторами проведена проверка погрешности экстарполяции при различных значениях тока в намагничивающей обмотке. Погрешность не превышает $1,1 \%$. 


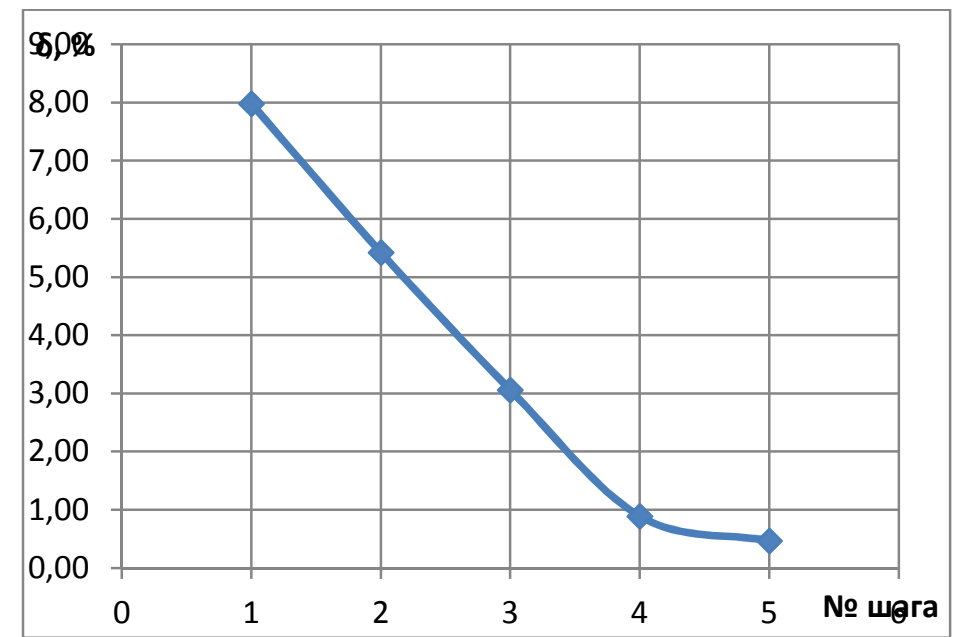

Рис. 5. Изменение погрешности при движении по градиенту

\section{Список литературы}

1. Адлер Ю.П., Маркова Е.В., Градовский Ю.В. Планирование эксперимента при поиске оптимальных условий. - М.: Наука, 1976. - 278 с.

2. Ланкин М.В., Кучер А.И. Устройство определения магнитных характеристик листовой электротехнической стали с каналом измерения напряженности магнитного поля// Теория, методы и средства измерений, контроля и диагностики: материалы XIII Междунар. науч.практ. конф. - Новочеркасск: ЮРГТУ(НПИ), 2012. - С. 11-14.

3. Пат. 100833 Российская Федерация, МПК G01R33/00. Устройство для измерения магнитных характеристик полос из листовой стали/ Шайхутдинов Д.В.; заявитель и патентообладатель Шайхутдинов Д.В. - №2010139473/28; заявл. 24.09.2010; опубл. 27.12.2010, Бюл. № 36 4. Халафян A.A. Statistica 6. Статистический анализ данных - М.: Бином-пресс, 2007. - 512 с. 5. Шкуратник В.Л. Измерения в физическом эксперименте -М.: Горная книга, 2006. - 335 с.

\section{Рецензенты:}

Горбатенко Н.И., д.т.н., профессор, И.О. первого проректора, ЮРГПУ(НПИ) им. М.И. Платова, г.Новочеркасск

Гречихин В.В., д.т.н., доцент, доцент кафедры ИИСТ, ЮРГПУ(НПИ) им. М.И. Платова, г.Новочеркасск. 